\title{
Establishment of incontinence-associated dermatitis rat models and assessment of the therapeutic effects of zinc oxide, painless skin protective film and silicone dressing
}

\author{
GUIYUAN CHEN ${ }^{1}$, LIQUAN HUANG ${ }^{1}$, YINGXUN CHEN ${ }^{2}$, SHUFENG ZHENG ${ }^{3}$, \\ LOUYING ZHU $^{4}$ and MINGXING DING ${ }^{5}$
}

\begin{abstract}
${ }^{1}$ Nursing Faculty, School of Medicine, Jinhua Polytechnic, Jinhua, Zhejiang 321007; ${ }^{2}$ Department of General Medicine, Jinhua Municipal Central Hospital; ${ }^{3}$ Department of Gastroenterology, Jinhua People's Hospital;

${ }^{4}$ Jinhua Center of Laboratory Animals, Jinhua Municipal Food and Drug Inspection Institute, Jinhua, Zhejiang 321000;

${ }^{5}$ Medical Molecular Biology Laboratory, School of Medicine, Jinhua Polytechnic, Jinhua, Zhejiang 321007, P.R. China
\end{abstract}

Received February 1, 2021; Accepted July 1, 2021

DOI: $10.3892 / \mathrm{etm} .2021 .10492$

\begin{abstract}
The aim of the present study was to construct incontinence-associated dermatitis (IAD) rat models and observe the therapeutic effects of zinc oxide, painless skin protective film and silicone dressing on IAD. A total of 54 rats were randomly divided into nine groups: i) Control group; ii) trypsin model group; iii) model + zinc oxide group; iv) model + painless skin protective film group; v) model + silicon dressing group; vi) synthetic urine combined with trypsin model group (joint model group); vii) joint model + zinc oxide group; viii) joint model + painless skin protective film group; and ix) joint model + silicone dressing group. A total of 4 days after applying the zinc oxide, protective film or silicon dressing intervention, IAD scores and $\mathrm{pH}$ values in skin tissues were examined. Skin tissues and blood samples were collected. Hematoxylin and eosin staining, immunohistochemical staining of major histocompatibility complex class II (MHC-II) and western blot analysis of MHC-II, NF- $\kappa$ B/p65, phosphorylated (p)-NF- $\kappa$ B/p65, STAT1 and p-STAT1 were carried out in skin tissue. Serum IFN- $\gamma$, IL-1 $\beta$, IL-2 and TNF- $\alpha$ levels were determined using ELISA. The results demonstrated that $\mathrm{IAD}$ scores and $\mathrm{pH}$ values were both higher in the model groups than the control, which were significantly ameliorated by silicone dressing. The skin
\end{abstract}

Correspondence to: Dr Mingxing Ding, Medical Molecular Biology Laboratory, School of Medicine, Jinhua Polytechnic, 1188 Wuzhou Street, Wucheng, Jinhua, Zhejiang 321007, P.R. China E-mail: mtd5tc@163.com

Abbreviations: IAD, incontinence-associated dermatitis; H\&E, hematoxylin and eosin

Key words: incontinence-associated dermatitis, zinc oxide, painless skin protective film, silicone dressing, major histocompatibility complex class II, NF- $\kappa \mathrm{B}, \mathrm{STAT} 1$ tissue structure of IAD rats both in trypsin model group and joint model group was severely damaged, the wounds were not covered by epidermis, and numerous inflammatory cell infiltrations were observed. After treatment, dermatitis was improved. Skin tissue from the trypsin and joint IAD models had higher MHC-II, NF- $\kappa$ B p65, p-NF- $\kappa$ B p65, STAT1 and p-STAT1 expression than controls, which was decreased by protective film and silicon dressing. Zinc oxide reduced NF- $\kappa$ B p65, p-NF- $\kappa$ B p65, STAT1 and p-STAT1 expression. However, no significant differences were observed in $\mathrm{NF}-\kappa \mathrm{B} / \mathrm{p}-\mathrm{NF}-\kappa \mathrm{B}$ ratio and STAT1/p-STAT1 ratio among groups. Furthermore, serum IFN- $\gamma$, IL-1 $\beta$, IL- 2 and TNF- $\alpha$ levels were significantly elevated in trypsin and joint IAD rats. The upregulation of these cytokines was significantly inhibited after all three treatments. Among the three treatment methods, silicone dressing had the best therapeutic effect. Thus, these findings revealed that zinc oxide, painless skin protective film and silicone dressing could ameliorate the severity of IAD rat models, and that silicone dressing possessed the best therapeutic effect.

\section{Introduction}

Incontinence-associated dermatitis (IAD) is inflammation of the skin caused by long-term exposure of the perineum or other skin to urine or feces (1). It is clinically manifested as erythema, with or without erosion or secondary infection (2). It widely occurs in bedridden patients, the elderly and critically ill patients $(3,4)$. Risk factors for IAD include incontinence chemical irritants (such as proteases and lipases that digest intestinal enzymes), changes in skin surface $\mathrm{pH}$ and associated micro-organisms (such as fungal infections caused by Candida albicans), repeated skin cleansing activities, and occluded perineal environment (such as the use of airtight nursing pads) and mechanical factors such as friction (5). IAD is recognized as a risk factor for pressure ulcers, causing serious inconvenience and pain to patients (6). Therefore, the development of preventive and therapeutic methods for IAD is urgently needed. 
Currently, the prevention and treatment of IAD include: i) Correcting the causes of diarrhea and incontinence; ii) reducing urine and stool irritation; iii) correct cleaning, moisturizing and skin care; iv) maintaining the skin $\mathrm{pH}$; and v) regular observation and evaluation (7-9). Since the presence of high moisture and corrosive enzymes in the intestinal juice can cause destructive damage to the skin, leading to peeling and erosion of the cortex, barrier products such as petrolatum, polydimethylsiloxane and zinc oxide ointment are necessary to protect patients with IAD (10). Although several studies on the prevention and treatment of IAD have been conducted, there is significant heterogeneity and low comparability between results (11-13). It is difficult for clinical medical staff to determine the relative performance of these barrier materials. Indeed, due to deficiencies in knowledge and clinical evidence, product selection is still a challenge faced by clinical medical staff when preventing and managing IAD. The aim of the present study was to identify a simple and easy-to-replicate rat model of IAD, and to study the effect of zinc oxide, painless skin protective film and silicone dressing on the healing of IAD and to provide experimental evidence for the treatment of IAD.

\section{Materials and methods}

Animals. 63 male Sprague-Dawley rats (weight, 150-220 g) aged 7-8 weeks were provided by The Jinhua Center of Laboratory Animals (Zhejiang, China). Animal experiments were carried out in The Jinhua Food and Drug Inspection and Testing Research Institute (Zhejiang, China). Before the experiment, all rats were adaptively fed for one week. All procedures were performed following the recommendations of the Guide for the Care and Use of Laboratory Animals of the National Institutes of Health. This study was approved by The Ethics Committee of The School of Medicine, Jinhua Polytechnic (approval no. 2019018).

Construction of an IAD model. In an initial experiment, 9 rats were randomly divided into three groups according to a random number table: i) Control group; ii) trypsin-induced IAD (model) group; and iii) synthetic urine combined with trypsin-induced IAD (joint model) group. The artificial urine was generated as follows: i) $25 \mathrm{~g}$ urea (purity $>99 \%$ ); ii) 9 g sodium chloride (>99\%); iii) 3 g ammonium chloride (>99.9\%); iv) 3 g sodium sulfite (>98\%); v) $2.5 \mathrm{~g}$ anhydrous disodium hydrogen orthophosphate (>99\%); and vi) $2 \mathrm{~g}$ creatinine (>99\%). These were dissolved in 1,000 ml Ultrapure water prepared using a Direct Q5 purification system (EMD Millipore). Subsequently, 25\% ammonium hydroxide solution was added into $25 \mathrm{ml}$ of the above mixture, thus resulting in synthetic urine solution with $1 \%$ ammonium hydroxide. Sodium hydroxide was added into $4 \mathrm{~g} / 100 \mathrm{ml}$ trypsin liquid or synthetic urine combined with trypsin, thus adjusting $\mathrm{pH}$ to 7.5-8.5.

The selected area on the back of the rat was covered with a cotton ball containing the trypsin solution or the synthetic urine with trypsin and adhesive tape (3M company) and fixed with an elastic bandage. The cotton ball was maintained for 4 days. Once a day in the morning and afternoon, $5 \mathrm{ml}$ corresponding solution was added to the cotton ball of the rat to keep the cotton ball moist and continuously covered the back of the rat. IAD scores and skin pH values were examined every day. After 4 days, the bandage was removed and the severity of IAD was observed, including the size of the dermatitis occurrence area and the IAD score of severity. The rats were anesthetized using an intraperitoneal injection of sodium pentobarbital. All procedures were strictly in line with The Guide for the Care and Use of Laboratory Animals of the National Institutes of Health. The control group was treated with cotton balls soaked in saline.

Assessment of the IAD model. According to the IAD severity assessment tool scale described by Borchert et al (14), the rats were observed and evaluated once a day before and after the intervention for 4 days. Furthermore, the degree of recovery from dermatitis was also evaluated after the intervention according to the severity of the rash and missing skin. IAD was evaluated based on the degree of skin redness in the back, skin loss and rash. The scoring criteria were as follows: i) None, 0 point; ii) erythema, 1 point; iii) edema, 2 points; iv) papule, 3 points; and v) erosion and superficial ulcer, 4 points.

Animal groups. After successful modeling, 54 rats were randomly divided into 9 groups according to the random number table ( $n=6$ in each group): i) Control group; ii) trypsin model group; iii) model + zinc oxide group; iv) model + painless skin protective film group; v) model + silicon dressing group; vi) synthetic urine combined with trypsin model group (joint model group); vii) joint model + zinc oxide (Guangzhou Baiyunshan Pharmaceutical Co., Ltd.) group; viii) joint model + painless skin protective film (3M company) group; and ix) joint model + silicone dressing (Jiangsu Youchuang Biomedical Technology Co., Ltd.) group.

For rats in the zinc oxide group, zinc oxide ointment was evenly applied $>1 \mathrm{~cm}$ away from the urine- and feces-contaminated skin, twice a day. For rats in the painless skin protective film group, the skin protective film was sprayed twice a day. The distance was $10-15 \mathrm{~cm}$ from the urine- and stool-contaminated skin and $1 \mathrm{~cm}$ beyond the urine and stool contaminated skin. The painless skin protective film was applied on the affected skin. For rats in the silicone dressing group, the silicone dressing was applied twice a day to the affected skin.

IAD severity assessment and skin $\mathrm{pH}$ test were performed for each group every 4 days after applying the zinc oxide, protective film or silicon dressing intervention. After the rats had been anesthetized by intraperitoneal injection of pentobarbital sodium $(60 \mathrm{mg} / \mathrm{kg})$, blood samples were collected from the eyeball. All rats were then sacrificed by intraperitoneal injection of an overdose of pentobarbital sodium $(200 \mathrm{mg} / \mathrm{kg})$. Skin tissue samples were then collected.

Hematoxylin and eosin $(H \& E)$ staining. Fresh skin tissue samples were fixed in $4 \%$ paraformaldehyde (Wuhan Google Biotechnology Co., Ltd.) for $24 \mathrm{~h}$ at $4^{\circ} \mathrm{C}$. After dehydration and paraffin embedding, tissue sections were cut to 4- $\mu \mathrm{m}$ thickness. After the paraffin sections were deparaffinized, H \& E staining was performed. The sections were stained with hematoxylin (cat. no. B600020; ProteinTech Group) at $37^{\circ} \mathrm{C}$ for $5 \mathrm{~min}$ and $0.5 \%$ eosin solution (Sigma-Aldrich; Merck KGaA) at $37^{\circ} \mathrm{C}$ for $2 \mathrm{~min}$. After dehydration, the sections were mounted with 
neutral gum and placed under a light microscope (BX53; Olympus Corporation) for observation.

Immunohistochemistry. The skin tissue sections were fixed with $4 \%$ paraformaldehyde overnight at $4^{\circ} \mathrm{C}$ and paraffin-embedded. The tissue sections were cut to $4 \mu \mathrm{m}$ thick and blocked with goat serum (Beyotime Institute of Biotechnology) for $30 \mathrm{~min}$ at room temperature. The sections were incubated with anti-major histocompatibility complex class II (MHC-II) antibody (1:200; cat. no. ab23990; Abcam) overnight at $4^{\circ} \mathrm{C}$, then with HRP-conjugated goat anti-mouse secondary antibody (1:200; cat. no. K5007; Dako; Agilent Technologies) for $30 \mathrm{~min}$ at room temperature. After the sections were stained with DAB for 5-10 min and the nuclei were counterstained by hematoxylin for 3-5 min. After dehydration and transparency, the sections were mounted with neutral gum (cat. no. G1403; Sangon Biotech Co., Ltd.). Finally, images were acquired and analyzed under an light microscope (Olympus Corporation). The Image-Pro Plus 6.0 (Media Cybernetics, Inc.) image processing system was used to measure the optical density of MHC-II protein in each group.

Western blot analysis. The tissue specimens were extracted with $200 \mu 1$ RIPA lysis buffer (Beyotime Institute of Biotechnology) at $4^{\circ} \mathrm{C}$ for $30 \mathrm{~min}$. After centrifugation at $12,000 \mathrm{x} \mathrm{g}$ for $10 \mathrm{~min}$ at $4^{\circ} \mathrm{C}$, the supernatant was collected and stored at $-80^{\circ} \mathrm{C}$. The BCA quantification kit was utilized for evaluating the protein concentration. A total of $30 \mu \mathrm{g}$ protein was loaded per lane and the samples were separated by $12 \%$ polyacrylamide gel electrophoresis, then transferred to a PVDF membrane. The membrane was blocked with $5 \%$ skimmed milk powder for $2 \mathrm{~h}$ at room temperature. The membrane was then incubated with primary antibodies against MHC-II (1:1,000; cat. no. ab23990; Abcam), $\beta$-actin (1:1,000; cat. no. ab179467; Abcam), NF-кB/p65 (Ser727; 1:1,000; cat. no. 10745-1-AP; ProteinTech group), phosphorylated (p)-NF-кB p65 (Ser536; 1:1,000; cat. no. 3033T; Cell Signaling Technology, Inc.), p-STAT1 (1:1,000; cat. no. ab126598; Abcam), STAT1 (1:1,000; cat. no. 10144-2-AP; ProteinTech group) and GAPDH (1:1,000; cat. no. 60004-1-Ig; ProteinTech group) overnight at $4^{\circ} \mathrm{C}$, then incubated with HRP-conjugated goat anti-mouse secondary antibody (1:5,000; cat. no. SA00001-1; ProteinTech group) at room temperature for $1.5 \mathrm{~h}$. ECL Plus Luminescence Kit (Beyotime Institute of Biotechnology) was used to visualize protein bands. ImageJ software (version 1.48; National Institutes of Health) was used to quantify protein expression levels.

ELISA. The blood samples from rat eyeball were allowed to stand for $30 \mathrm{~min}$ at room temperature. After centrifugation at $3,500 \times \mathrm{g}$ for $15 \mathrm{~min}$ at $4^{\circ} \mathrm{C}$, the serum samples were obtained. IFN- $\gamma$ (cat. no. RK00199), IL-1 $\beta$ (cat. no. RK00009), IL-2 (cat. no. RK00010) and TNF- $\alpha$ (cat. no. RK00029) ELISA kits (all from ABclonal Biotech Co., Ltd.) were separately used to quantify IFN- $\gamma$, IL-1 $\beta$, IL-2 and TNF- $\alpha$ levels in serum.

Statistical analysis. SPSS 18.0 software was used for statistical analysis. The data were expressed as the mean \pm standard deviation. For IAD severity, Kruskal-Wallis followed by Dunn's post hoc test was performed. For all other variables, one-way
ANOVA followed by Tukey's post hoc test was used. $\mathrm{P}<0.05$ was used to indicate a statistically significant difference.

\section{Results}

Construction of IAD models. In the present study, trypsin-induced IAD and synthetic urine combined with trypsin-induced IAD models were constructed. H\&E staining results showed that the skin structure of rats in the control group was complete and clear. The skin tissue structure of the rats in the trypsin-induced model group and the synthetic urine combined with the trypsin model (joint model) group was severely damaged (Fig. 1).

Zinc oxide, painless skin protective film and silicone dressing decrease IAD score and $p H$ of IAD rats. A total of 54 rats were randomly divided into nine groups, as described in Materials and methods. All rats were carefully observed each day (Fig. 2). The present study found that there was significant dermatitis in IAD rats compared to rats in the control group. However, after treatment with zinc oxide, painless skin protective film or silicone dressing, the dermatitis of IAD rats was significantly ameliorated. Following treatment for 4 days, the IAD scores and $\mathrm{pH}$ values of the rats were obtained. As shown in Fig. 3A, compared with rats in the control group (average IAD score $=0.333$ ), the severity of dermatitis in IAD rats induced by trypsin (model group; average IAD score $=3 ; \mathrm{P}<0.01$ ) or artificial urine combined with trypsin (joint model group; average IAD score=3.333; $\mathrm{P}<0.001$ ) was significantly increased. Compared with the model group, dermatitis significantly improved after 4 days of treatment with silicone dressing (average IAD score; $\mathrm{P}<0.05$ ). However, zinc oxide (average IAD score $=2 ; \mathrm{P}>0.05$ ) and painless skin protective film (average IAD score $=1.667 ; \mathrm{P}>0.05$ ) did not reduce the severity of dermatitis. Compared with the joint model group, dermatitis significantly improved after 4 days of treatment with painless skin protective film (the average value of $\mathrm{IAD}=0.833 ; \mathrm{P}<0.05$ ) or silicone dressing (average IAD score $=0.667 ; \mathrm{P}<0.01$ ), but not with zinc oxide (average IAD score $=1.667 ; \mathrm{P}>0.05$ ). The therapeutic effect of silicon dressing was the largest of the three interventions.

Furthermore, the $\mathrm{pH}$ of rat skin was also tested (Fig. 3B). In model and joint model groups, the $\mathrm{pH}$ value of rat skin was significantly higher than that of the control group, and the difference was statistically significant (both $\mathrm{P}<0.0001$ ). Treatment with zinc oxide, painless skin protective film or silicone dressing significantly reduced the $\mathrm{pH}$ value of the skin in both models (all $\mathrm{P}<0.0001$ ).

Zinc oxide, painless skin protective film and silicone dressing improve dermatitis in IAD rat models. After treatment with zinc oxide, painless skin protective film or silicone dressing for 4 days, skin tissue samples were taken for observation. H\&E staining was used to detect the pathological changes associated with dermatitis. As shown in Fig. 4, compared with rats in the control group, the skin tissue structure in both model groups was severely damaged. After 4 days of treatment with zinc oxide, painless skin protective film or silicone dressing, the skin tissues showed distinct epidermal and dermal structures; however, each group still had different degrees of hair follicle 


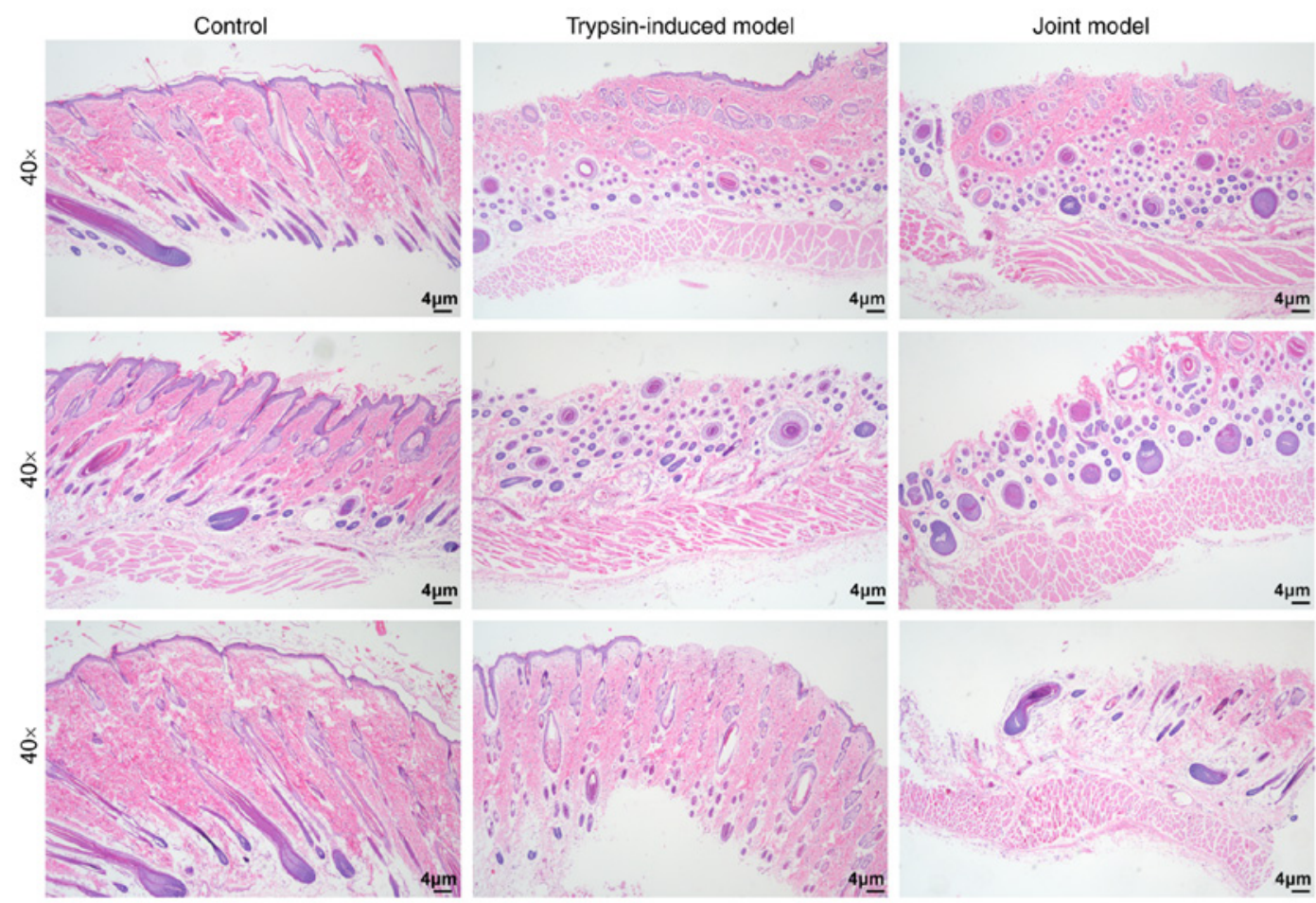

Figure 1. Construction of IAD rat models. Hematoxylin and eosin staining was used to assess the pathological changes in skin tissue samples from the control, trypsin-induced IAD model and synthetic urine combined with trypsin-induced IAD model groups. Magnification, $\mathrm{x} 40$. IAD, incontinence-associated dermatitis.

damage and edema. Among them, rats in the silicone dressing treatment group displayed the best recovery.

Zinc oxide, painless skin protective film and silicone dressing lower expression of MHC-II in IAD rat models. Immunohistochemistry and western blot experiments were utilized to detect the expression of MHC-II in rat skin tissues. In Fig. 5A-C, there was almost no expression of MHC-II in the skin tissues of rats in the control group. The expression of MHC-II in the skin tissues of the trypsin model group and the artificial urine combined with trypsin model group were significantly increased compared to the control group. Furthermore, the expression of MHC-II in the combined model group was slightly higher than that of the trypsin model group. Compared with the model group, the expression of MHC-II in the zinc oxide, painless skin protective film, and silicone treatment groups all decreased to varying degrees, and the decrease was the most significant in the silicone dressing treatment group.

Zinc oxide, painless skin protective film and silicone dressing do not decrease the $N F-\kappa B / p-N F-\kappa B$ and STAT1/p-STATI ratios in IAD rat models. Western blotting was used to detect

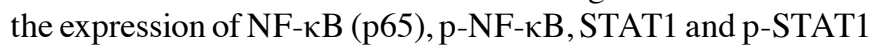
in rat skin tissue (Fig. 6A). Compared with the control group, the expression levels of NF- $\kappa \mathrm{B}(\mathrm{P}<0.0001$; Fig. $6 \mathrm{~B}), \mathrm{p}-\mathrm{NF}-\kappa \mathrm{B}$ $(\mathrm{P}<0.0001$; Fig. 6C), STAT1 $(\mathrm{P}<0.0001$; Fig. 6D) and p-STAT1 $(\mathrm{P}<0.0001$; Fig. 6E) were higher in the skin tissues of the trypsin model group and the artificial urine combined with the trypsin model group. However, compared to the control group, no significant differences in $\mathrm{NF}-\kappa \mathrm{B} / \mathrm{p}-\mathrm{NF}-\kappa \mathrm{B}$ ratio (Fig. $6 \mathrm{~F}$ ) or STAT1/p-STAT1 ratio (Fig. 6G) were observed in the trypsin model group or the artificial urine combined with the trypsin model group, suggesting that phosphorylation levels of
$\mathrm{NF}-\kappa \mathrm{B}$ and STAT1 were not affected in the two IAD models. After treatment with painless skin protective film $(\mathrm{P}<0.001$ or $\mathrm{P}<0.01$ ) or silicone dressing (both $\mathrm{P}<0.0001$ ), the expression of $\mathrm{NF}-\kappa \mathrm{B}$ significantly decreased in the skin tissues of the trypsin model and the artificial urine combined with the trypsin model (Fig. 6B). However, zinc oxide did not change NF- $\kappa$ B expression in the skin tissues of the IAD models. p-NF- $\kappa$ B levels in the skin tissues of the trypsin model and the combined model was significantly lowered by painless skin protective film (both $\mathrm{P}<0.001$ ) and silicone dressing (both $\mathrm{P}<0.0001$ ). Moreover, zinc oxide decreased the expression of $\mathrm{p}-\mathrm{NF}-\kappa \mathrm{B}$ in the skin tissues of the trypsin model $(\mathrm{P}<0.05)$, but not the combined model (Fig. 6C). STAT1 and p-STAT1 expression in the skin tissues of IAD rats was significantly reduced by zinc oxide, painless skin protective film and silicone dressing (Fig. 6D and E). Among them, the reduction levels of $\mathrm{NF}-\kappa \mathrm{B}$, $\mathrm{p}-\mathrm{NF}-\kappa \mathrm{B}, \mathrm{STAT} 1$ and $\mathrm{p}-\mathrm{STAT} 1$ were the most significant in the silicon dressing treatment group. However, no significant differences were found in $\mathrm{p}-\mathrm{NF}-\kappa \mathrm{B} / \mathrm{NF}-\kappa \mathrm{B}$ ratio (Fig. $6 \mathrm{~F}$ ) or p-STAT1/STAT1 ratio (Fig. 6G) in any group, suggesting that zinc oxide, painless skin protective film and silicone dressing did not affect the phosphorylation levels of NF- $\kappa$ B and STAT1 in IAD rat models.

Zinc oxide, painless skin protective film and silicone dressing decrease IFN- $\gamma, I L-1 \beta, I L-2$ and TNF- $\alpha$ levels in serum samples of IAD rats. ELISA was performed to examine the serum levels of IFN- $\gamma$, IL-1 $\beta$, IL-2 and TNF- $\alpha$. Compared with the control group, serum IL-1 $\beta$ (Fig. 7A), IL-2 (Fig. 7B), IFN- $\gamma$ (Fig. 7C) and TNF- $\alpha$ (Fig. 7D) levels were significantly elevated in both model groups (all $\mathrm{P}<0.0001)$. After treatment with zinc oxide, painless skin protective film and silicone dressing, their serum levels were significantly reduced in IAD models. 


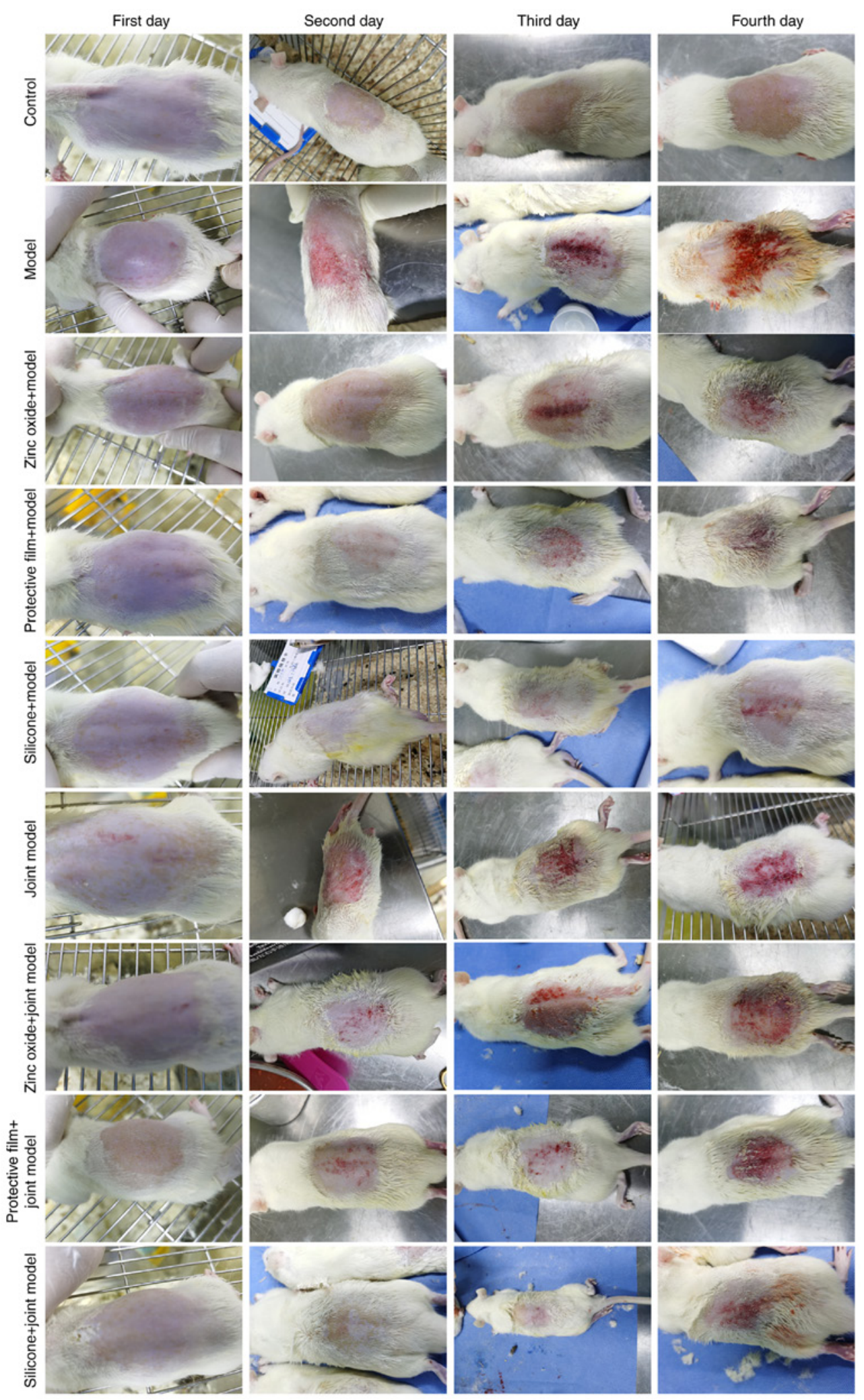

Figure 2. Photographs of the nine experimental groups on day 1,2,3 and 4 after treatment. There was significant dermatitis in trypsin-induced and synthetic urine combined with trypsin-induced IAD rats compared to rats in the control group. Following treatment with zinc oxide, painless skin protective film or silicone dressing for 1, 2,3 and 4 days, the dermatitis of IAD rats was significantly ameliorated. IAD, incontinence-associated dermatitis.

\section{Discussion}

IAD has a high incidence in the community, nursing homes and long-term care institutions, as well as in the clinical departments and intensive care units of hospitals. It has severely reduced the patients' quality of life and increased the economic burden of patients (15). Therefore, the development of preventive and therapeutic methods for IAD is urgently needed. In the present study, rat models of IAD were established using two methods (trypsin model and synthetic urine combined with trypsin 

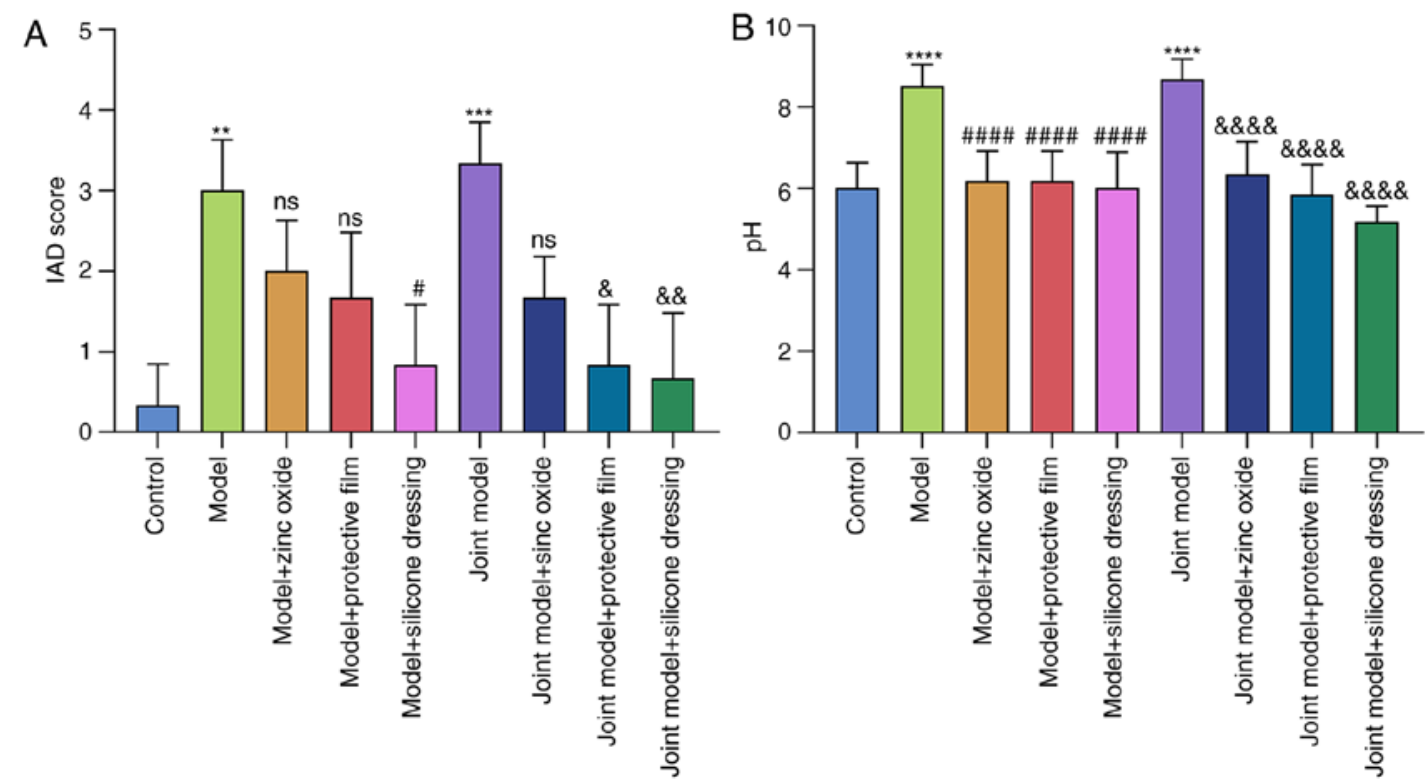

Figure 3. Effects of zinc oxide, painless skin protective film and silicone dressing on the IAD score and skin pH of IAD rats induced by trypsin or synthetic urine combined with trypsin. (A) Assessment of IAD scores in each group. (B) Determination of $\mathrm{pH}$ values in each group. ${ }^{* *} \mathrm{P}<0.01,{ }^{* * * *} \mathrm{P}<0.001$,

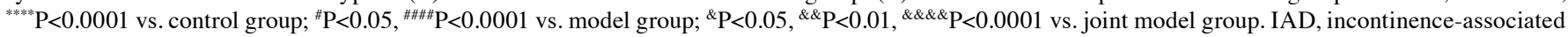
dermatitis; ns, not significant.

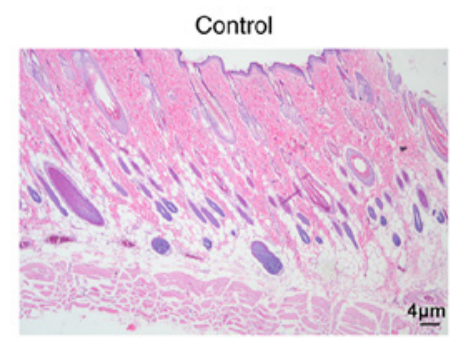

Painless skin protective film+ trypsin-induced IAD

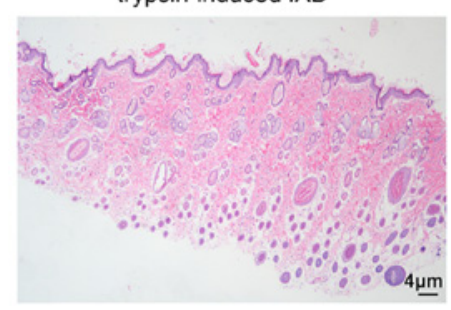

Zinc oxide+IAD joint model

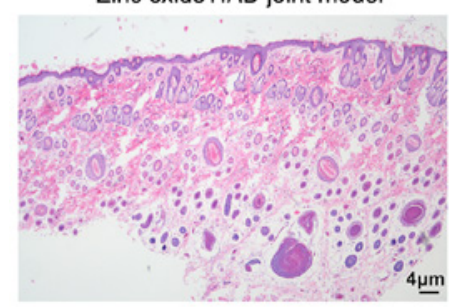

Trypsin-induced IAD

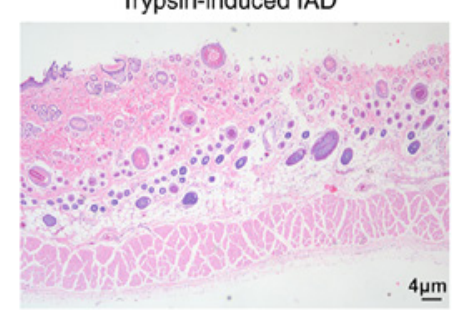

Silicone dressing+trypsin-induced IAD

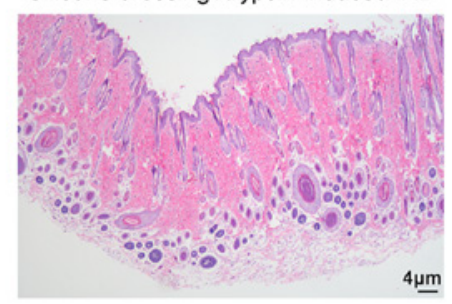

Painless skin protective film + IAD joint model

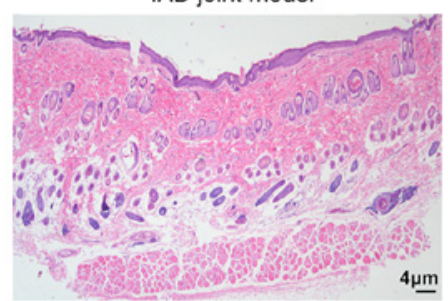

Zinc oxide+trypsin-induced IAD

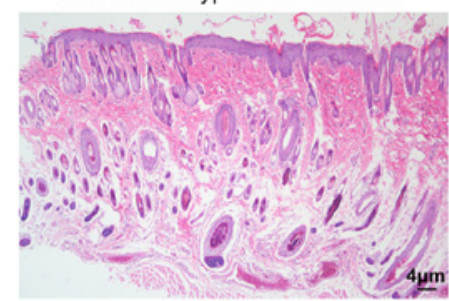

IAD joint model

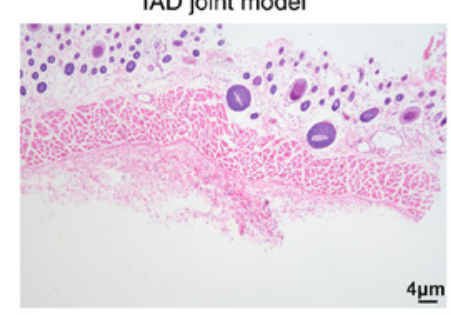

Silicone dressing +IAD joint model

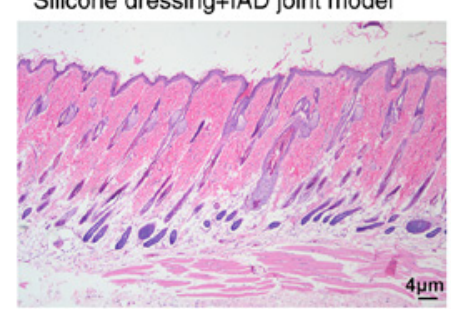

Figure 4. Hematoxylin and eosin staining for the effects of zinc oxide, painless skin protective film and silicone dressing on dermatitis of IAD rats. Magnification, x40. IAD, incontinence-associated dermatitis.

model). The effects of zinc oxide, painless skin protective film and silicone dressing on these IAD models were then examined.

There are still very few animal models of IAD. As early as 2011, Minematsu et al (16) used agar gel to soak the soles of the feet of aged rats, which provides a potential method to construct IAD animal models. Been et al (17) established an IAD model by immersion and fixation of $1 \%$ pancreatin on the back of rats. Mugita et al (18) 
A

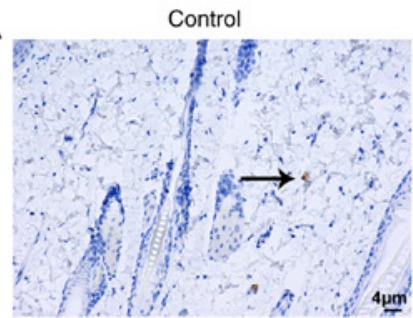

Painless skin protective film+ trypsin-induced IAD

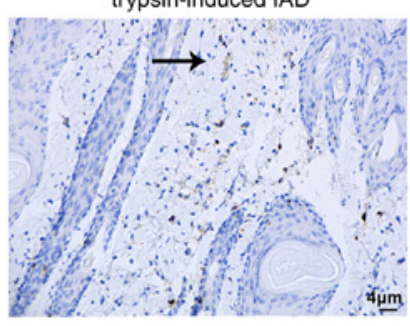

Zinc oxide+IAD joint mode

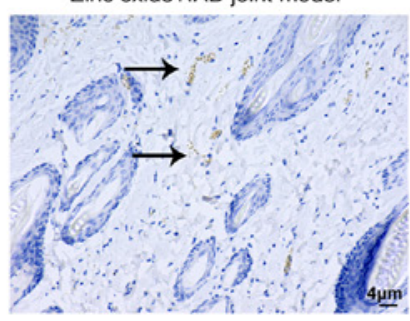

Trypsin-induced IAD

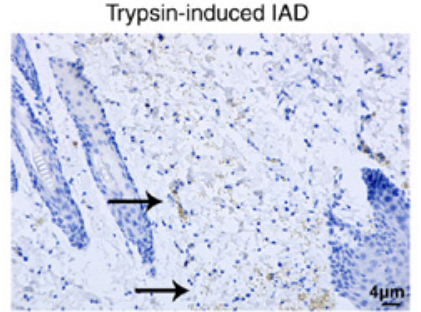

Silicone dressing+trypsin-induced IAD

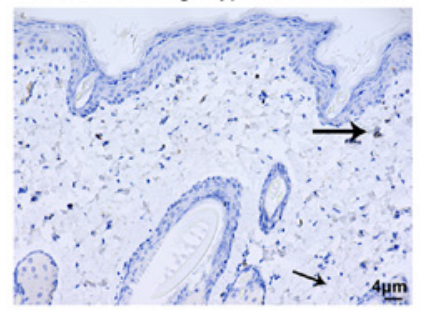

Painless skin protective film+

IAD joint model

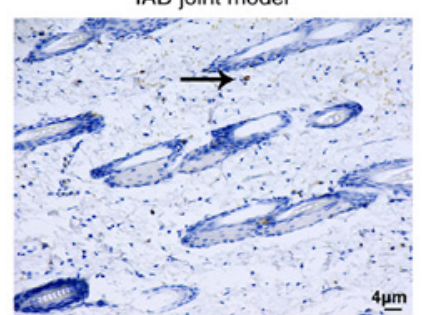

Zinc oxide+trypsin-induced IAD

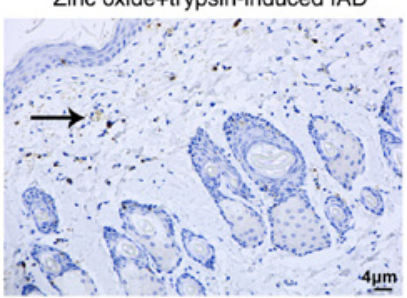

IAD joint model

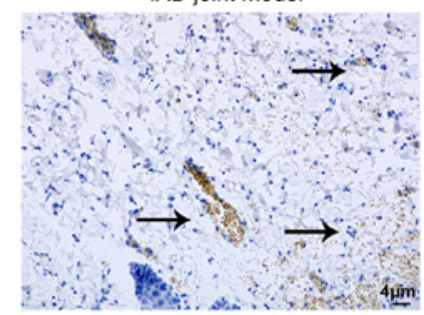

Silicone dressing+IAD joint model

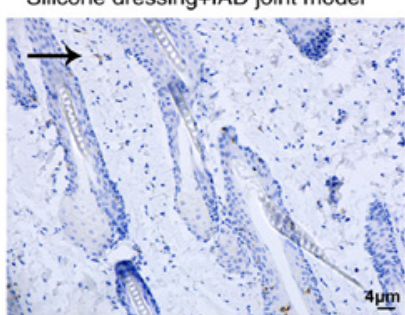

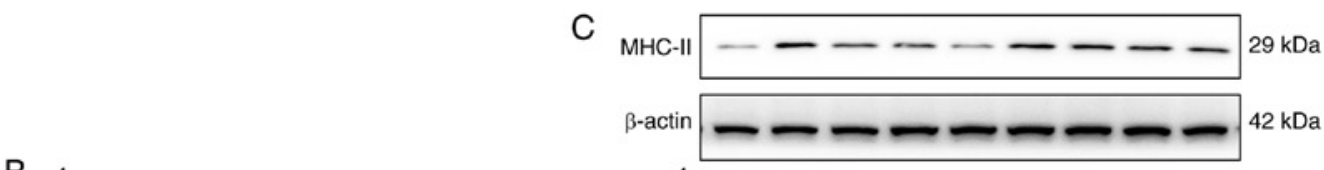
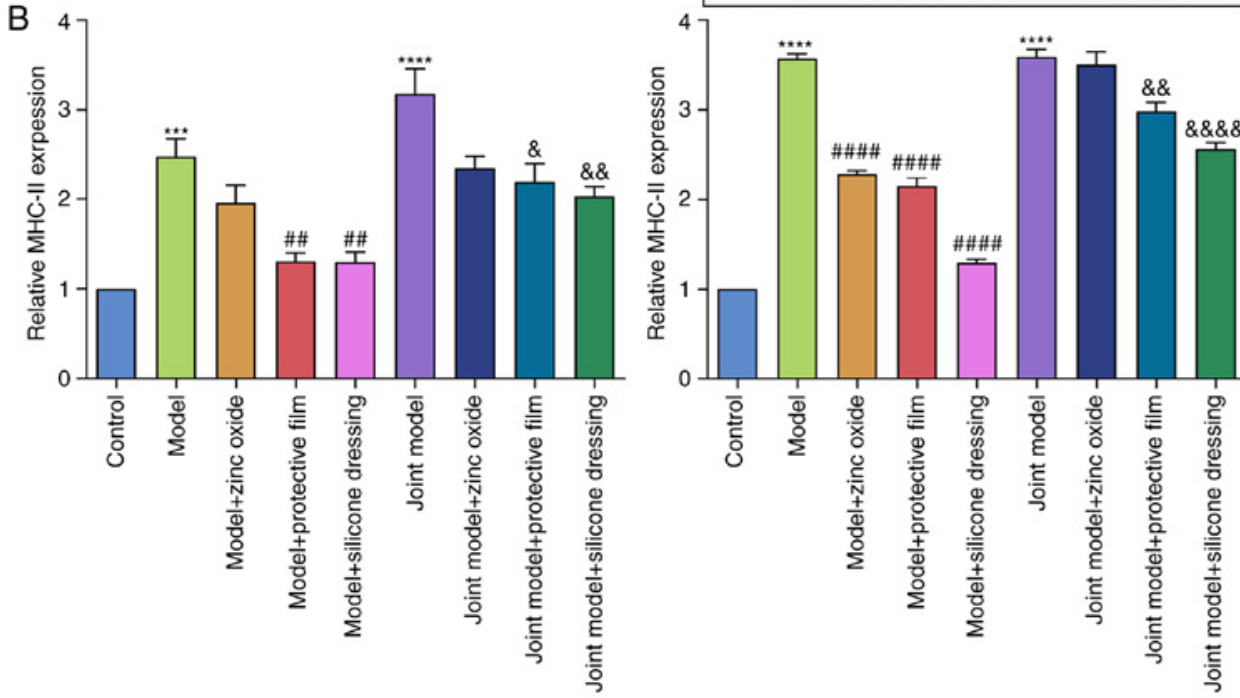

Figure 5. Evaluation of MHC-II expression in the skin tissue of rats in the control, trypsin-induced IAD model and synthetic urine combined with trypsin-induced IAD model groups. (A and B) Immunohistochemical detection of MHC-II expression in skin tissue samples from each group. Magnification, x200. MHC-II expression is indicated by the arrows. (C) Western blot analysis of MHC-II expression in the skin tissue from each group. ${ }^{* * *} \mathrm{P}<0.001,{ }^{* * * * *} \mathrm{P}<0.0001$ vs. control group; ${ }^{\# \#} \mathrm{P}<0.01,{ }^{\# \# \#} \mathrm{P}<0.0001$ vs. model group; ${ }^{\&} \mathrm{P}<0.05$, ${ }^{\&}{ }^{\&} \mathrm{P}<0.01$, ${ }^{\& \& \& \&} \mathrm{P}<0.0001$ vs. joint model group. MHC-II, major histocompatibility complex class II; $\mathrm{IAD}$, incontinence-associated dermatitis.

applied protease-containing agarose gel to the back skin of male SD rats for $4 \mathrm{~h}$, followed by inoculation with Pseudomonas aeruginosa for $30 \mathrm{~min}$. Wen et al (19) established an IAD model on the back of guinea pigs by using different concentrations of pancreatin solution, confirming that increased pancreatic juice concentration may lead to more severe skin reactions and higher IAD scores. In addition, Biçer et al (20) performed an ileostomy in rats to establish an ileostomy-related dermatitis model and used ozone to treat the resulting dermatitis. 

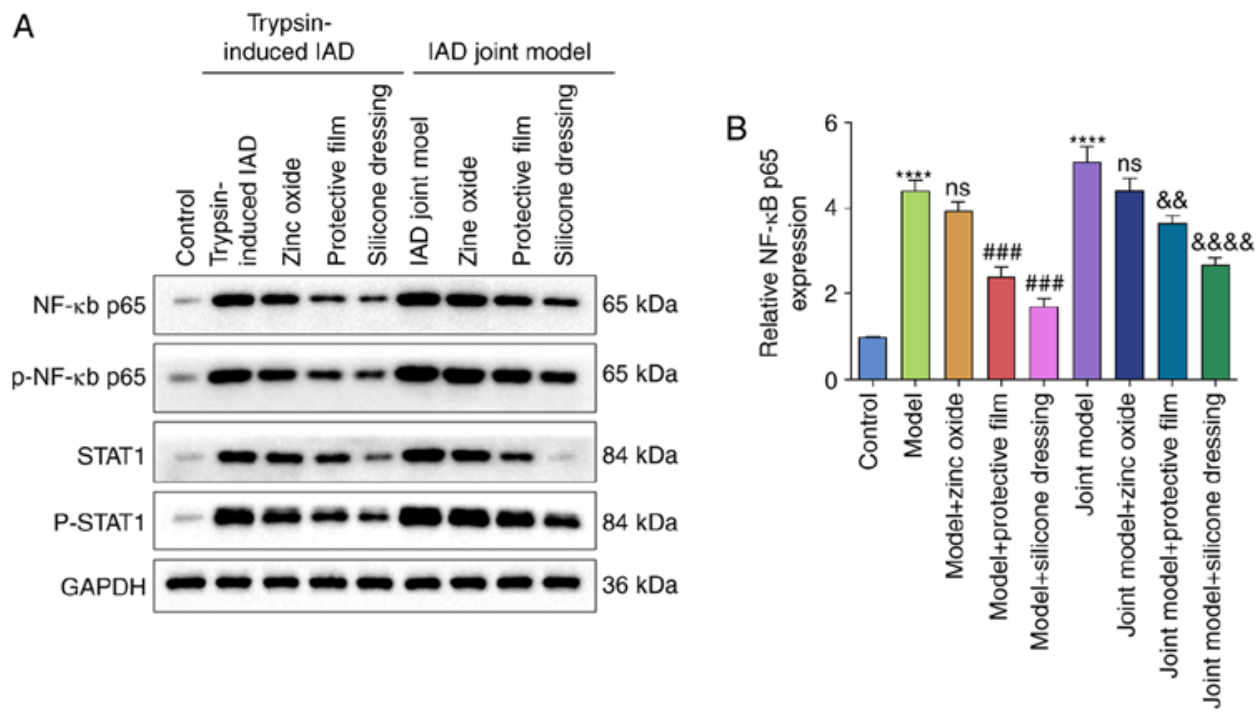
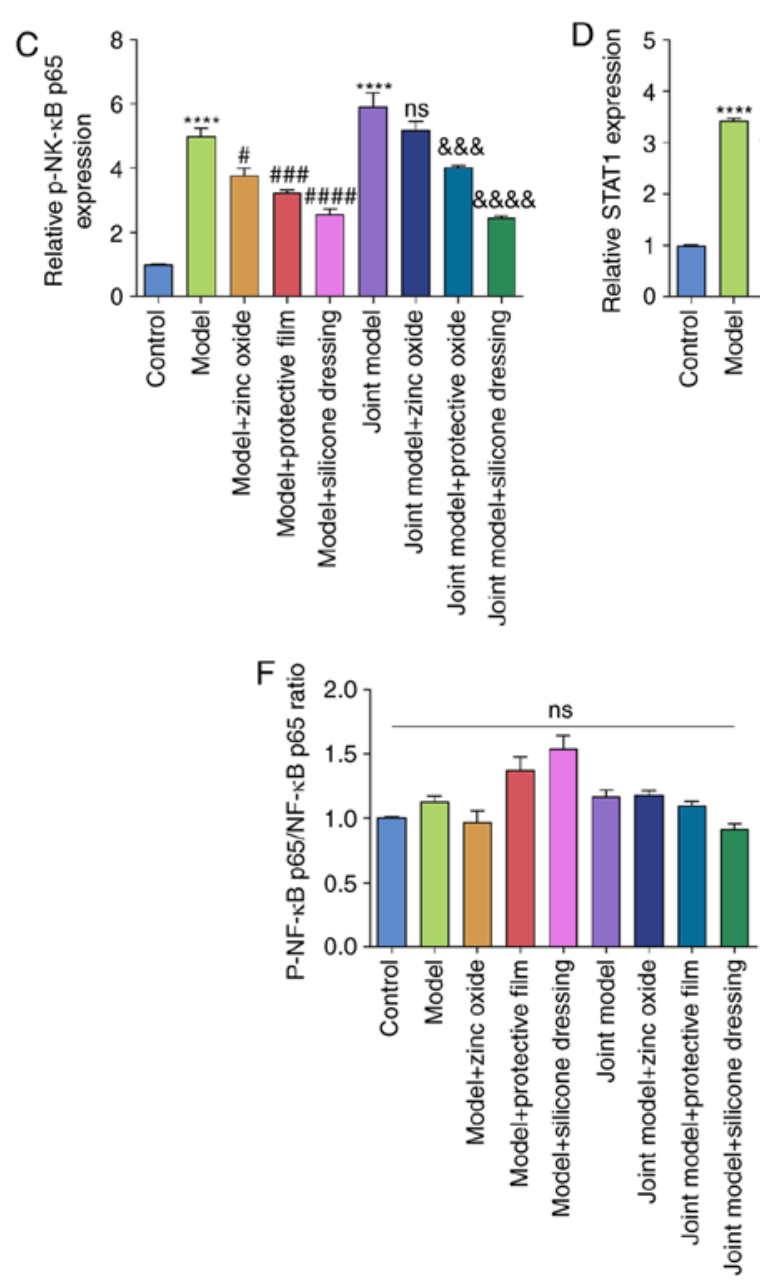
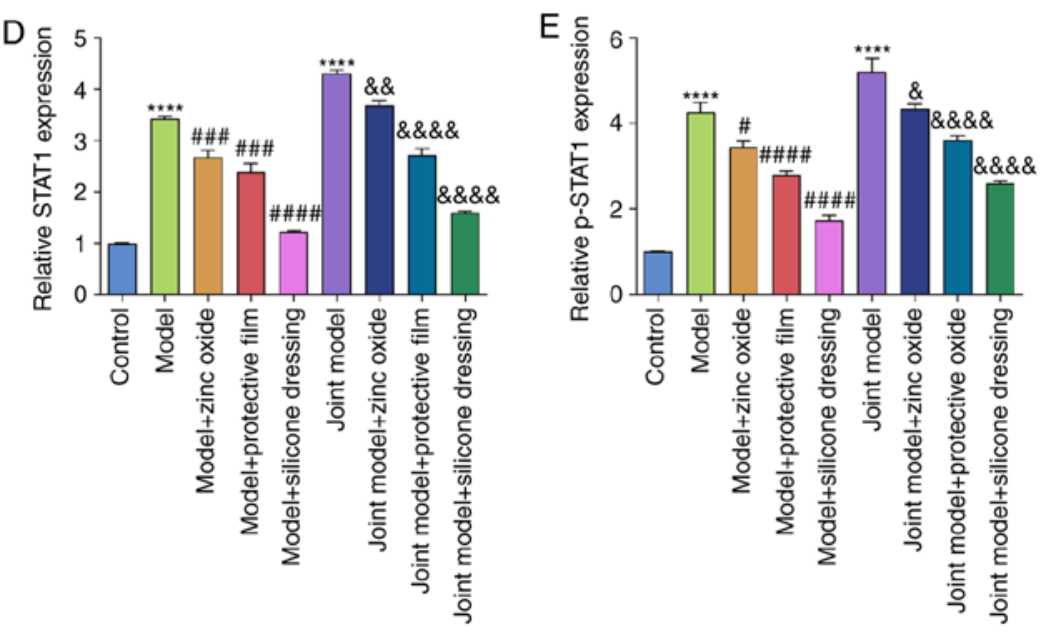

G

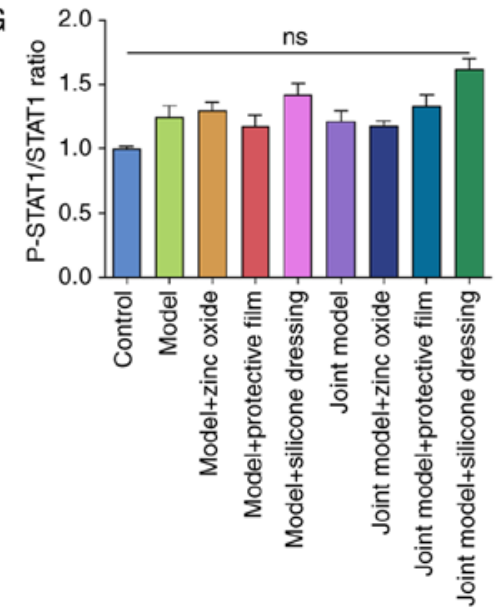

Figure 6. Western blot analysis of the expression of NF- $\mathrm{B} / \mathrm{p} 65, \mathrm{p}-\mathrm{NF}-\mathrm{kB} / \mathrm{p} 65$, STAT1 and p-STAT1 in the skin tissue of rats from the control, trypsin-induced IAD model and synthetic urine combined with trypsin-induced IAD model groups. (A) Representative images of western blots of each

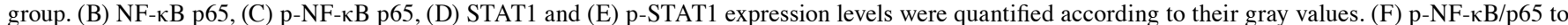

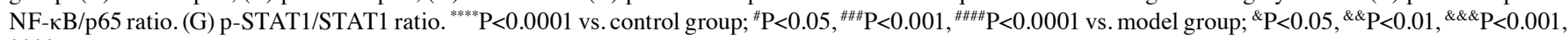
\&\&\&\&P<0.0001 vs. joint model group. IAD, incontinence-associated dermatitis; ns, not significant; $\mathrm{p}$-, phosphorylated.

The search for a simple and easy-to-replicate IAD animal model requires continual exploration. The skin is the body's protective layer that provides an important anatomical barrier to pathogens, irritants, moisture loss and environmental threats. Incontinence can damage the integrity of the epidermis due to excessive water, high $\mathrm{pH}$ and the presence of enzymes in the stool, leading to inflammation and pain and increasing the risk of infection (21). The cortex is affected by the external 
A

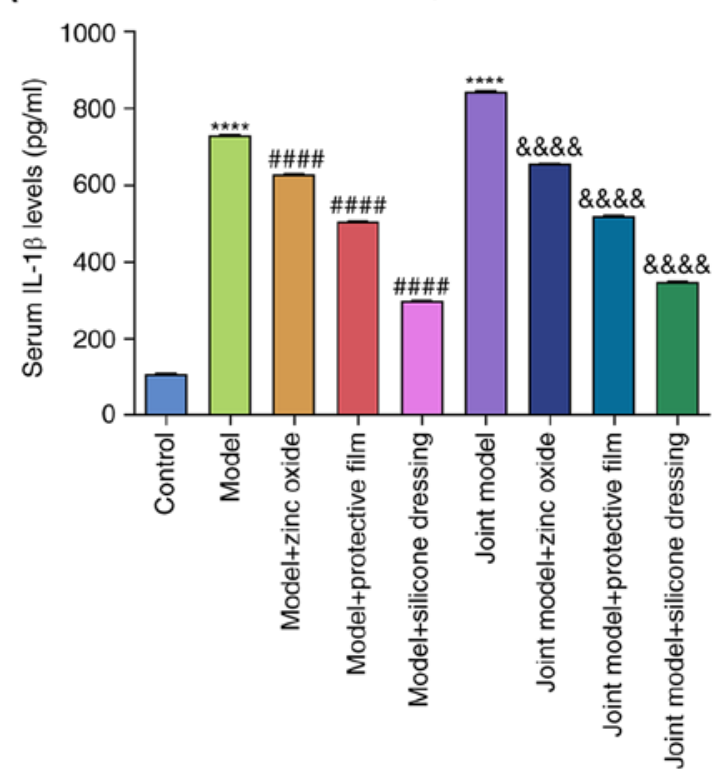

B

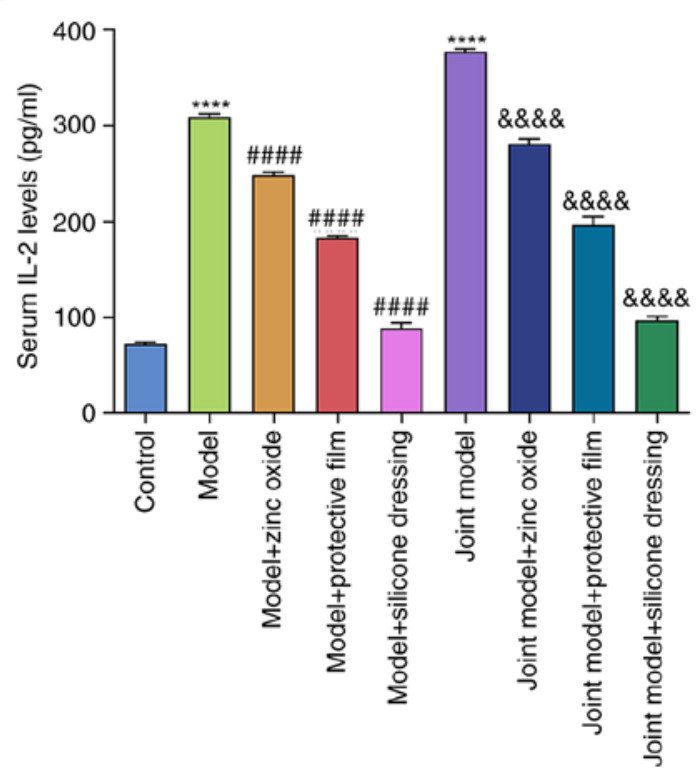

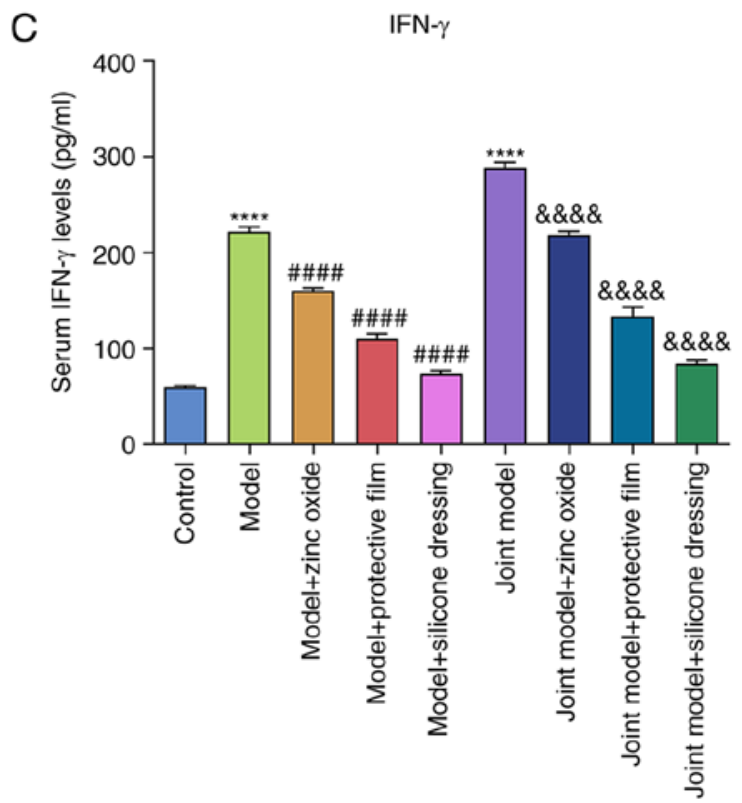

D

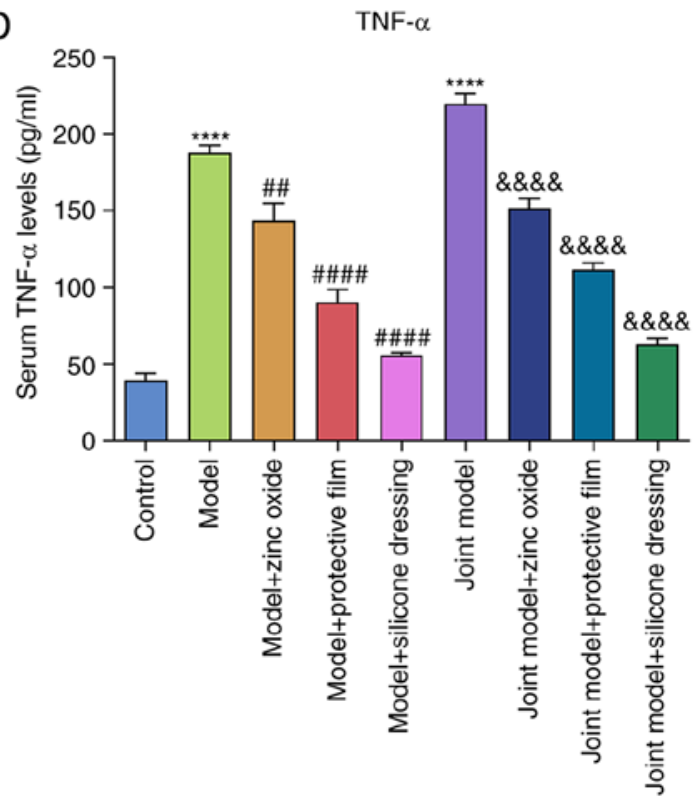

Figure 7. ELISA of the serum levels of IFN- $\gamma$, IL-1 $\beta$, IL-2 and TNF- $\alpha$ in the control, trypsin-induced IAD model and synthetic urine combined with trypsin-induced IAD model groups. (A) IL-1 $\beta$, (B) IL-2, (C) IFN- $\gamma$ and (D) TNF- $\alpha$ levels were examined in each group using ELISA. ${ }^{* * * *}$ P $<0.0001$ vs. control group, ${ }^{\# \#} \mathrm{P}<0.01,{ }^{\# \# \# \#} \mathrm{P}<0.0001$ vs. model group; ${ }^{\text {\&\&\&\& }} \mathrm{P}<0.0001$ vs. joint model group. IAD, incontinence-associated dermatitis.

environment, causing the cells to actively release related inflammatory mediators $(10,22)$. The keratinocytes in the cortex become increasingly less elastic, the cells shrink and the barrier function of the cortex is also weakened (23). Skin related damages such as loss, erosion, exudation and blisters appear in the cortex, which can be accompanied by bacterial or fungal infections (24). In addition, long-term skin contact with urine or feces causes excessive moisture on the cortex to overwhelm the stratum corneum cortex structure, and exhibit excessive hydration or maceration (25). When the digestive enzymes present in the feces encounter the urea present in the urine, they are used to produce repeated cycles of chemical stimulation, leading to inflammation and decomposition of the cortex $(9,26)$. In the present study, two methods were used to construct the IAD rat model. H\&E staining confirmed the establishment of dermatitis in both models. These two IAD models are simple to implement and are worth studying further.

IAD is one of the most common problems in clinical nursing. There is no uniform treatment standard for IAD, but choosing the right skin care program is key to identifying one. In the present study, after 4 days of treatment with zinc oxide, painless skin protective film or silicone dressing, dermatitis was significantly relieved and skin $\mathrm{pH}$ was significantly reduced. H\&E staining results indicated that the skin tissues significantly recovered following treatment. Park (27) found that silicone dressings can effectively reduce the incidence of pressure ulcers in patients with IAD and improve the symptoms of dermatitis. In the present study, silicone dressing had the most visible therapeutic effect on IAD. MHC-II plays 
an important role in the pathogenesis of dermatitis (27-29). Inhibiting the expression of NF- $\kappa$ B p65, p-NF- $\mathrm{B}$ p65, STAT1 and p-STAT1 can effectively reduce the severity of dermatitis (30-32). Compared with the control group, the expression of MHC-II, NF- $\kappa$ B p65, p-NF- $\kappa$ B p65, STAT1 and p-STAT1 in the skin tissues of the model groups increased significantly. After treatment, the expression of the above proteins was significantly reduced. The reduction in the silicone dressing group was the most apparent. These results indicated that zinc oxide, painless skin protective film and silicone dressings can effectively inhibit inflammation-related pathways in IAD, thereby improving the symptoms of dermatitis. Further studies are needed to examine the molecular mechanisms of zinc oxide, painless skin protective film or silicone dressings in the treatment of IAD.

In conclusion, zinc oxide, painless skin protective film and silicone dressings significantly ameliorated dermatitis in rats with IAD. Among these treatment modalities, silicone dressings exhibited the best therapeutic effects. Thus, these intervention methods warrant further validation.

\section{Acknowledgements}

Not applicable.

\section{Funding}

This work was funded by The Basic Public Welfare Research Program of Zhejiang Province (grant no. LGD20C040002) and The Science and Technology Project of Jinhua City in China (grant no. 2019-4-074).

\section{Availability of data and materials}

The datasets used and/or analyzed during the current study are available from the corresponding author on reasonable request.

\section{Authors' contributions}

MD conceived and designed the study. GC and LH conducted most of the experiments and data analysis and wrote the manuscript. YC, SZ and LZ conducted a number of experiments and data analysis, and contributed to the writing and revision of the manuscript. MD and GC confirm the authenticity of all the raw data. All authors read and approved the final manuscript.

\section{Ethics approval and consent to participate}

The study was approved by The Ethics Committee of The School of Medicine, Jinhua Polytechnic (approval no. 2019018).

\section{Patient consent for publication}

Not applicable.

\section{Competing interests}

The authors declare that they have no competing interests.

\section{References}

1. Beele H, Smet S, Van Damme N and Beeckman D: Incontinenceassociated dermatitis: Pathogenesis, contributing factors, prevention and management options. Drugs Aging 35: 1-10, 2018.

2. Koudounas S, Bader DL and Voegeli D: Knowledge gaps in the etiology and pathophysiology of incontinence-associated dermatitis: A scoping review. J Wound Ostomy Continence Nurs 47: 388-395, 2020.

3. Raepsaet C, Fourie A, Van Hecke A, Verhaeghe S and Beeckman D: Management of incontinence-associated dermatitis: A systematic review of monetary data. Int Wound J 18: 79-94, 2021.

4. Zhang Y, Leng M, Guo J, Duan J and Wang Z: The effectiveness of faecal collection devices in preventing incontinence-associated dermatitis in critically ill patients with faecal incontinence: A systematic review and meta-analysis. Aust Crit Care 34: 103-112, 2021.

5. Lichterfeld-Kottner A, El Genedy M, Lahmann $N$, Blume-Peytavi U, Büscher A and Kottner J: Maintaining skin integrity in the aged: A systematic review. Int J Nurs Stud 103: $103509,2020$.

6. Barakat-Johnson M, Basjarahil S, Campbell J, Cunich M, Disher G, Geering S, Ko N, Lai M, Leahy C, Leong T, et al: Implementing best available evidence into practice for incontinence-associated dermatitis in Australia: A multisite multimethod study protocol. J Tissue Viability 30: 67-77, 2021.

7. Gates BP, Vess J, Long MA and Johnson E: Decreasing incontinence-associated dermatitis in the surgical intensive care unit: A quality improvement project. J Wound Ostomy Continence Nurs 46: 327-331, 2019.

8. Kottner J, Hahnel E, El Genedy M, Neumann K and Balzer K: Enhancing SKIN health and safety in aged CARE (SKINCARE Trial): A study protocol for an exploratory cluster-randomized pragmatic trial. Trials 20: 302, 2019.

9. Phipps L, Gray M and Call E: Time of onset to changes in skin condition during exposure to synthetic urine: A prospective study. J Wound Ostomy Continence Nurs 46: 315-320, 2019.

10. Acton $\mathrm{C}$, Ivins N, Bainbridge $\mathrm{P}$ and Browning P: Management of incontinence-associated dermatitis patients using a skin protectant in acute care: A case series. J Wound Care 29: 18-26, 2020.

11. Van Damme N, Van Hecke A, Himpens A, Verhaeghe S and Beeckman D: Design and psychometric testing of the attitude towards the prevention of incontinence-associated dermatitis instrument (APrIAD). Int Wound J 16: 492-502, 2019.

12. Gray M: Context for practice: Prevention of pressure injury and incontinence-associated dermatitis. J Wound Ostomy Continence Nurs 44: 406-408, 2017.

13. Kayser SA, Phipps L, VanGilder CA and Lachenbruch C: Examining prevalence and risk factors of incontinence-associated dermatitis using the international pressure ulcer prevalence survey. J Wound Ostomy Continence Nurs 46: 285-290, 2019.

14. Borchert K, Bliss DZ, Savik K and Radosevich DM: The incontinence-associated dermatitis and its severity instrument: Development and validation. J Wound Ostomy Continence Nurs 37: 527-535, 2010.

15. Arnold-Long M and Johnson E: Epidemiology of incontinence-associated dermatitis and intertriginous dermatitis (Intertrigo) in an acute care facility. J Wound Ostomy Continence Nurs 46: 201-206, 2019.

16. Minematsu T, Yamamoto Y, Nagase T, Naito A, Takehara K, Iizaka S, Komagata K, Huang L, Nakagami G, Akase T, et al: Aging enhances maceration-induced ultrastructural alteration of the epidermis and impairment of skin barrier function. J Dermatol Sci 62: 160-168, 2011.

17. Been RA, Bernatchez SF, Conrad-Vlasak DM, Asmus RA, Ekholm BP and Parks PJ: In vivo methods to evaluate a new skin protectant for loss of skin integrity. Wound Repair Regen 24: 851-859, 2016.

18. Mugita Y, Minematsu T, Huang L, Nakagami G, Kishi C, Ichikawa Y, Nagase T, Oe M, Noguchi H, Mori $\mathrm{T}$, et al: Histopathology of incontinence-associated skin lesions: Inner tissue damage due to invasion of proteolytic enzymes and bacteria in macerated rat skin. PLoS One 10: e0138117, 2015.

19. Wen Z, Zhu W, Liu Q, Zhang H, Mei B and Shen M: Development of an animal model for inducing various degrees of severity of incontinence-associated dermatitis. J Wound Ostomy Continence Nurs 44: 578-582, 2017.

20. Biçer Ş, Sayar I, Gürsul C, Işı1k A, Aydın M, Peker K and Demiryilmaz I: Use of ozone to treat ileostomy dermatitis in an experimental rat model. Med Sci Monit 22: 757-765, 2016. 
21. Hoedl M and Eglseer D: Which characteristics of fecal incontinence predispose incontinence-associated dermatitis? A classification and regression tree analysis. Adv Skin Wound Care 34: 103-108, 2021

22. Koudounas S, Mugita Y, Minematsu T, Nakagami G, Weller C and Sanada H: Does the presence of bacterial urinary infection contribute to the development of incontinence-associated dermatitis? A scoping review. J Tissue Viability 30: 256-261, 2021

23. Hödl M, Blanař V, Amir Y and Lohrmann C: Association between incontinence, incontinence-associated dermatitis and pressure injuries: A multisite study among hospitalised patients 65 years or older. Australas J Dermatol 61: e144-e146, 2020.

24. Coyer F, Campbell J and Doubrovsky A: Efficacy of incontinence-associated dermatitis intervention for patients in intensive care: An open-label pilot randomized controlled trial. Adv Skin Wound Care 33: 375-382, 2020.

25. Tay C, Yuh AS, Sheau Lan EL, Ong CE, Aloweni F and Lopez V: Development and validation of the incontinence associated dermatitis knowledge, attitude and practice questionnaire. J Tissue Viability 29: 244-251, 2020.

26. Werth SL and Justice R: Prevalence of moisture-associated skin damage in an acute care setting: Outcomes from a quality improvement project. J Wound Ostomy Continence Nurs 46: 51-54, 2019.

27. Park KH: The effect of a silicone border foam dressing for prevention of pressure ulcers and incontinence-associated dermatitis in intensive care unit patients. J Wound Ostomy Continence Nurs 41: 424-429, 2014
28. Aquino M and Rosner G: Systemic contact dermatitis. Clin Rev Allergy Immunol 56: 9-18, 2019.

29. Kolesnik M, Franke I, Lux A, Quist SR and Gollnick HP: Eczema in psoriatico: An important differential diagnosis between chronic allergic contact dermatitis and psoriasis in palmoplantar localization. Acta Derm Venereol 98: 50-58, 2018.

30. Gil TY, Kang YM, Eom YJ, Hong CH and An HJ: Anti-atopic dermatitis effect of seaweed fulvescens extract via inhibiting the STAT1 pathway. Mediators Inflamm 2019: 3760934, 2019.

31. Yang BY, Cheng YG, Liu Y, Liu Y, Tan JY, Guan W, Guo S and Kuang HX: Datura Metel L. Ameliorates imiquimod-induced psoriasis-like dermatitis and inhibits inflammatory cytokines production through TLR7/8-MyD88-NF- $\mathrm{BB}-\mathrm{NLRP} 3$ Inflammasome pathway. Molecules 24: 2157, 2019.

32. Irrera N, Vaccaro M, Bitto A, Pallio G, Pizzino G, Lentini M, Arcoraci V, Minutoli L, Scuruchi M, Cutroneo G, et al: BAY 11-7082 inhibits the NF- $\mathrm{kB}$ and NLRP3 inflammasome pathways and protects against IMQ-induced psoriasis. Clin Sci (Lond) 131: 487-498, 2017.

This work is licensed under a Creative Commons Attribution-NonCommercial-NoDerivatives 4.0 International (CC BY-NC-ND 4.0) License. 